\title{
Measuring health behaviour and health: towards new health promotion indicators
}

\author{
HORST NOACK
}

Department of Social and Preventive Medicine, University of Berne, Switzerland

\section{INTRODUCTION}

In a growing number of countries efforts are being made to improve the health of the people by adopting new approaches to health promotion and the prevention of ill health. In 1984, the Member States of the European Region of the World Health Organization (WHO) endorsed 38 regional targets as a part of the regional strategy for health for all (WHO, 1985).

In November 1986 over 200 participants from 38 countries attended the first International Conference on Health Promotion in Ottawa, Ontario, Canada, and discussed the needs for and steps towards a new public health. The Ottawa Charter for Health Promotion (1986), which has received wide public and professional attention, identifies five main kinds of action for health promotion: building healthy public policy, creating supportive environments, strengthening community action, developing personal skills and reorienting health services. Health promotion action means advocacy for health, enabling people to achieve their full health potential, and mediating between different interests in the pursuit of health. Although the Ottawa Conference focused on health promotion strategies for industrialized countries, similar concerns in other countries were taken into account.

In countries that are planning or already have introduced health promotion and disease prevention programmes, a strong need is felt to develop and test new measurements or indicators for evaluating programmes and monitoring change. In addition to national and regional indicators that are thought to be suitable for the assessment of progress towards important health targets (macro-level indicators), sufficiently sensitive instruments are needed to measure changes in important conditions of and processes related to health and changes in specific dimensions of health (micro-level indicators).

It has been said that a first task is to identify new indicators, in particular in the fields of lifestyles, the quality of life and health equity, in order to assess the relationship between those factors and health status. At present, there are too many traditional indicators such as those of mortality; by focusing more on means than on ends, and more on the providers than on the users of health care, the present indicators are out of line with the main thrust of the regional strategy for health for all.

To assist Member States, WHO (1981) published a book that explains what health indicators are and why they are needed. This publication summarizes global or macro-level indicators of social and economic development, health care provision, coverage by primary health care, and basic health status, as well as mortality, morbidity and wellbeing. In the volume Targets for health for all (WHO, 1985), both essential and optional indicators are listed that are thought to enable systematic monitoring and the evaluation of progress in each Member State and in the Region as a whole.

WHO has also initiated and supported activities undertaken by organizations and individuals within the research community. Measurement in health promotion and protection (Abelin et al., 1987), published under the joint sponsorship of WHO and the International Epidemiological Association, contains a number of relevant 
conceptual and methodological papers written by epidemiologists and social scientists from many countries.

In several workshops held in the United Kingdom, ecologists, epidemiologists, sociologists and health statisticians from several European countries reviewed some of the more recent research on health indicators, assessed research needs and made suggestions for future directions of work (Culyer, 1983). At a conference in the United States on health services research, the current state of measuring health status was analysed and several relevant dimensions of health were discussed (Bergner, 1985).

Two recent conferences addressed fundamental issues in the measurement of health and healthrelated quality of life. An article on the Portugal Conference (Katz, 1987) reported how medical, behavioural and social scientists examined the progress that has been made in "the science of quality of life" and the role that measurements of the quality of life play for a broad range of decisions in the life of chronically ill people, in clinical practice, and in programme planning and policy-making. These scientists also identified remaining challenges. At the Advances in Health Assessment Conference (Lohr \& Ware, 1987), held in February 1986 in Palm Springs, California, both developers and users of health assessment methods discussed conceptual and methodological issues, and the application of health assessment tools to biomedical research, clinical practice and the assessment of health policy.

As the reports on these conferences indicated, there have been significant advances in research on the quality of life and in the development of reliable and valid measures of health and its various dimensions. The health-related quality of life, or what has come to be labelled, somewhat inappropriately, health status, may be an important aim or, if reliably measured, the crucial criterion of health promotion. Referring to the conditions and processes that maintain or improve the health-related quality of life or health status, however, the concept of health promotion has an entirely different meaning. To assess or measure health promotion per se requires different measures or indicators.

The Workshop on Measuring Health Behaviour and Health: towards New Health Promotion Indicators, held in Berne, and the work undertaken since then represents only a first step: namely, to make a critical review of the state of the art, to identify potential candidates for health promotion indicators, and to help establish a network for communication and collaboration.

\section{HEALTH TARGETS AND HEALTH PROMOTION}

The major purpose of measuring health-related processes and dimensions of health is, of course, to study or evaluate the health situation of a given population or population group in respect of certain health goals. There are good reasons to assume that the regional targets for health for all (WHO, 1985) represent a widely accepted set of health goals for the industrialized world. Of these 38 targets, five are particularly relevant to health promotion.

Target 13 calls for healthy public policy:

By 1990, national policies in all Member States should ensure that legislative, administrative and economic mechanisms provide broad intersectoral support and resources for the promotion of healthy lifestyles and ensure effective participation of the people at all levels of such policymaking.

Target 14 focuses on social support systems:

By 1990, all Member States should have specific programmes which enhance the major roles of the family and other social groups in developing and supporting healthy lifestyles.

Target 15 is concerned with knowledge and motivation for healthy behaviour:

By 1990 , educational programmes in all Member States should enhance the knowledge, motivation and skills of people to acquire and maintain health.

Target 16 calls for the encouragement of positive health behaviour:

By 1995, in all Member States, there should be significant increases in positive health behaviour, such as balanced nutrition, nonsmoking, appropriate physical activity and good stress management.

Target 17 calls for a decline in health-damaging behaviour:

By 1995, in all Member States, there should be significant decreases in health-damaging behaviour, such as overuse of alcohol and pharma- 
ceutical products; use of illicit drugs and dangerous chemical substances; and dangerous driving and violent social behaviour.

What does the concept of health promotion as the key strategy directed towards these targets mean? The WHO Working Group on Concepts and Principles of Health Promotion (WHO, 1984) defined health promotion as "the process of enabling people to increase control over, and to improve, their health", and health as a "resource for everyday life, not the objective of living; ... . a positive concept emphasizing social and personal resources, as well as physical capacities".

As a unifying concept health promotion is discussed in terms of five main principles (WHO, 1984):

- Health promotion involves the population as a whole in the context of their everyday life, rather than focusing on people at risk for specific diseases...

- Health promotion is directed towards action on the determinants or causes of health . . .

- Health promotion combines diverse, but complementary, methods or approaches, including communication, education, legislation, fiscal measures, organizational change, community development and spontaneous local activities against health hazards.

- Health promotion aims particularly at effective and concrete public participation. This focus requires the further development of problemdefining and decision-making lifeskills both individually and collectively.

- While health promotion is basically an activity in the health and social fields, and not a medical service, health professionals-particularly in primary health care-have an important role in nurturing and enabling health promotion...

The Working Group proposed that health promotion should focus on access to health by reducing inequalities in health and by increasing opportunities to improve health. First, this will require environments conducive to health, particularly at work and in the home. Second, because social forces are critical determinants of health-related values and behaviour it will be important to strengthen social networks and social support. Third, because of their potential benefit to health, positive health behaviour and appropriate coping strategies need to be promoted. Fourth, in order to provide a basis for making informed choices, it is necessary to increase the public's knowledge of health and to disseminate relevant information.

The Working Group also recognized several political and moral dilemmas in health promotion. Thus, there is a danger that health may be viewed as the ultimate goal of life. As a consequence, certain health-related practices may be prescribed to individuals rather than deliberately chosen through informed consent. Quite often health promotion will inappropriately focus on individuals' behaviour rather than on the social and economic conditions of health. Further, health information and other relevant health resources are frequently not easily accessible. Finally, there is a real possibility that certain professional groups will dominate health promotion to the exclusion of other professional groups and lay people.

\section{A CONCEPTUAL FRAMEWORK}

Population-centred programmes for health promotion and disease prevention are viewed here as interventions in a complex socioecological system, aiming at specific health-related changes within the system. Of course, changes in health are known to take place without any specific intervention. The question is whether and to what degree observed changes in health-related processes or in dimensions of health can be attributed to a particular intervention.

In general it is far easier to identify the phenomena to be measured and to define, in practical terms, the health variables of interest if there is consensus on a suitable conceptual framework. For the purpose of the Berne Workshop a framework was chosen that delineates five groups of relevant concepts and hence five groups of potential measurements or indicators: healthy public policy and health promotion programmes; societal and community health resources; group and personal health resources; health-related social processes and behaviour; and dimensions of health (Fig. 1).

\section{Healthy public policy}

Healthy public policy includes legal measures and regulations introduced to protect the population against health hazards and to maintain or develop an environment conducive to health. For 


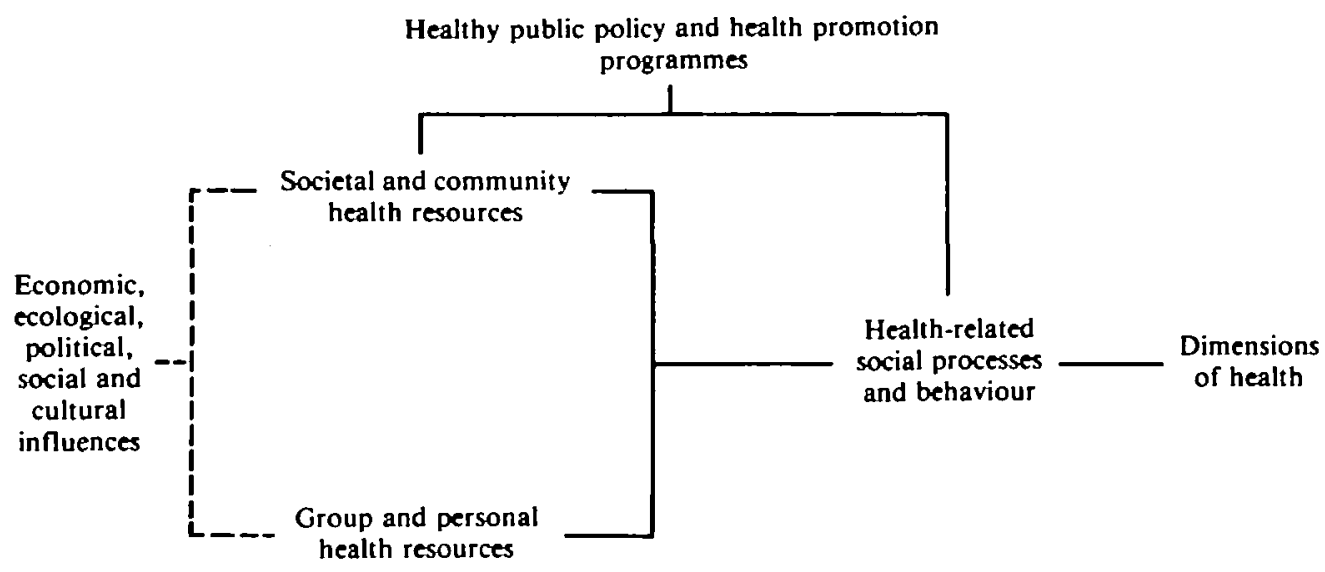

Fig. 1: Conceptual framework of health-related conditions and processes and of dimensions of health

example, such a policy may attempt to restrict the distribution of harmful products and promote those that are good for health, or it may attempt to disseminate health information through the mass media and intensify health education in schools, at the workplace and in primary care institutions. As part of healthy public policy, specific health promotion programmes may be implemented that are directed towards changes in lifestyle and people's active involvement in health-related activities.

A number of indicators were proposed to assess and compare health policies and health promotion programmes at the national and international level (Abelin et al., 1987; Hunt et al., 1986; McDowell \& Newell, 1987; WHO, 1985). Examples are indicators such as the existence of laws and regulations on the safety and labelling of goods, the taxation and advertising of healthdamaging products, the time allocated to health information programmes on television, the coordination and integration of health promotion activities in government or the community, and public participation in health activities at community or regional levels.

According to the proposed conceptual framework (Fig. 1), healthy public policy and specific health promotion programmes are assumed to influence the health resources of societies and communities and of groups and individuals as well as health-related social processes and behaviours. It is important to note that health resources may represent both relatively static elements (such as shared health knowledge, and cultural values and practices) and more or less dynamic elements (such as health information campaigns, and the use of preventive medical services).

\section{Societal and community health resources}

Societal and community health resources comprise a large variety of factors such as environmental conditions (housing, clean air and water, and access to healthful products or goods), economic factors (work, income and social security), social conditions (social contact and integration, social support and caring), and cultural conditions (shared health-related values, health knowledge and health-enhancing practices). Further important resources are government and nongovernmental bodies that initiate and support: health promotion programmes, educational institutions disseminating health information and teaching health-related skills, primary care and other medical institutions offering preventive services, or self-help and other lay groups involved in health promotion activities. Again, numerous measurements or indicators have been proposed or can be easily defined (Abelin et al., 1987).

\section{Group and personal health resources}

Group and personal health resources include the health-related rules, norms or customs shared by families and other primary social groups, the social relationships of members of these groups as well as people's physical characteristics (such as body mass, fitness and resistance to health 
hazards) and their psychological characteristics (such as behavioural and cognitive skills, and emotional stability). Many existing indicators can be used to assess these factors, both at the group and at the individual level (Abelin et al., 1987; WHO, 1985). Examples are: stable and supportive social networks, positive health values and attitudes in the family and other primary social groups, and adequate personal health knowledge and skills.

\section{Health-related social processes and behaviour}

Health-related social processes and behaviour refers to a set of phenomena that are distinct from, yet closely related to, health resources. In principle, all forms of interaction between a person and the environment that have a direct or indirect bearing on health belong to this category. Social behaviour and processes are very often seen as aspects of lifestyle.

An important category of these phenomena includes what is known or presumed to be positive health-related, or health-enhancing, behaviour. Well known examples are behaviour patterns such as eating a healthy diet, regular physical activity, coping effectively with strains in everyday life and at work, seeking reliable health information and using preventive health services. Another important category includes behaviour known to be health-damaging, particularly smoking, the abuse of alcohol and drugs, inadequate coping with stress, dangerous driving and violent social behaviour such as child abuse and wife-beating (Berkman \& Breslow, 1983; WHO, 1985).

It is important to note that almost all healthenhancing and health-damaging behaviour patterns are learned and hence perpetuated or changed through social learning processes throughout life. Further, many of these kinds of behaviour are not simply individual but collective or social in nature. A third important category, therefore, are the social processes that influence health indirectly (through affecting either healthenhancing or health-damaging behaviour) or directly (through changing psychological and physiological susceptibility or resistance). Social processes that were shown to have a significant indirect and/or direct influence on health are social and emotional support and caring.

A number of indicators or measurements of health-related social processes and behaviour are used in research and evaluation studies, especially at a global level or macro-level. Examples are the role and function of the family and other primary social groups, the proportion of people with or without regular social contact, the average daily intake of calories and important nutrients, the proportion of nonsmokers and regular smokers in a population, or average alcohol consumption per capita. Nevertheless, other studies are urgently needed: for conceptual clarification; for the development of suitable working models; for the identification of sensitive, reliable and valid measurements; and for the coordination of research. This is especially the case in research on health-enhancing and coping behaviour, and health-related social interaction.

\section{Dimensions of health}

Dimensions of health are a set of phenomena closely interrelated with health-related social processes and behaviour. Although these phenomena are frequently subsumed under the notion of health status, it is preferable to conceptualize health in terms of dynamic concepts such as health balance and health potential (Noack, 1987).

Several dimensions of dynamic health concepts can be considered. Thus, from a positive point of view, health balance often means biopsychosocial wellbeing, or an acceptable way of functioning as a whole person. Alternatively, a distinction may be made between physical, mental and social wellbeing or functioning. In terms of negative health, health balance frequently means an absence of symptoms, disease and disability.

Apart from dynamic balance, health can be viewed as health potential, in other words, as the capacity of a person or social group to maintain such a balance and to re-establish it when it is lost or threatened. Two important conditions need to be distinguished. One is a permanent process of physiological, psychological and behavioural health monitoring through, for example, specific health-related actions and coping techniques. The other important condition is the opportunity to make use of such a capacity. This may be possible only under particular environmental conditions, such as adequate housing and nutrition, a low level of health risks and sufficient social support.

Traditional health indicators or measurements largely reflect aspects of negative health, either specific disturbances of physical, mental or social functioning (morbidity, disability); or, 
more importantly, death rates for all causes of death, as well as for specific causes (mortality). Though there are measurements of a number of negative and positive dimensions of health (Bergner, 1985; Carley, 1981; Hansluwka, 1985; Mootz, 1986; Read et al., 1987), the challenge for the future is to develop instruments and indicators that are sensitive to changes in positive health in specific groups and populations. For example, it would be useful to have suitable measurements of changes in biopsychosocial wellbeing, physical and psychosocial functioning, or of changes in perceived symptoms and disability. It may also be helpful to be able to assess aspects of the health potential of groups and individuals, such as the capacity for selfhelp, coping competence or health expectations.

\section{SOME GENERAL CONSIDERATIONS IN INDICATOR DEVELOPMENT}

With the key concepts and variables identified within a conceptual framework (Fig. 1), it will be useful to consider the meaning of and the interrelationships of health-related indicators or measurements within the context of a suitable theoretical model. Two different types of models may be distinguished: the input-output model and the process model.

In the input-output model, independent variables (such as a given health policy, a health promotion programme, or certain health resources) are treated as inputs into the system, and changes or differences in health behaviour or dimensions of health in groups, as outputs. The social and behavioural processes involved in this type of model are perceived as a black box, and the nature of the processes involved and the transformation of input into output are not further analysed. Study design, measurement techniques and the statistical properties of measurements are considered crucial, as the primary goal is to make quantitative predictions or, at least, to identify changes or differences in outcome variables. The weakness of the input-output model is that it can hardly explain why changes do or do not occur, particularly when a suitable substantive theory is lacking (a very common situation in health research).

Process models, on the other hand, differ in focus. Rather than predicting outcomes, the major thrust is to describe, understand and explain the contextual, behavioural and biological processes involved in phenomena such as health behaviour, potential or balance. Within such a framework, it may be important to study, for example, the interrelationships between social values and social control, on the one hand, and health-enhancing or health-damaging behaviour, on the other. The difficulty with process models in general, and with more specific models of behavioural change in particular, is that they are particular research models rather than elaborated theories. A common misunderstanding requires clarification, however. Although process models tend to favour symbolic and qualitative representations of the phenomena studied, this certainly does not preclude the application of measurement techniques and quantitative analysis.

Which theoretical model or paradigmatic view and, as a consequence, which methodological approach may be the most fruitful in health indicator development will, of course, depend upon the purpose of a project and the questions to be answered. The purpose of a given project will further determine the kind of health and health promotion indicators needed. Broadly speaking, two different purposes can be distinguished; quantitative and qualitative healthrelated information can be used in scientific research and in practical or applied work. In the latter field, health-related information may be used to formulate health policies and to plan health promotion programmes; to evaluate such policies and programmes; or to monitor changes in health resources, health-related processes or behaviour and dimensions of health.

Any well planned project for indicator development requires, of course, that a number of specific steps be taken. The following sequence may serve as a rough guideline:

- specifying the purpose of the project and giving good reasons for it;

- identifying and describing the health-related phenomena to be assessed;

- defining and/or selecting the most suitable measurements or indicators;

- analysing the validity, reliability, sensitivity and cost of the selected measurements, using all available research results and experience; and

- choosing an ongoing study or planning a new one, in order to investigate the validity of 
measurements or indicators and other important properties.

As this last point suggests, it is strongly recommended that health indicators be developed and tested in the context of appropriate studies, such as national projects to monitor health and health promotion programmes based in the community or the workplace.

\section{REFERENCES}

Abelin, T. et al., ed. (1987). Measurement in health promotion and protection. Copenhagen, WHO Regional Office for Europe (WHO Regional Publications, European Series, No. 22).

Bergner, M. (1985). Measurement of health status. Medical care, 23: 696-704.

Berkman, L.F. \& Breslow, L. (1983). Health and ways of living. New York, Oxford University Press.

Carley, M. (1981). Social measurement and social indicators. London, Allen \& Unwin.

Culyer, A.J., ed. (1983). Health indicators. An international study for the European Science Foundation. Oxford, Martin Robertson.

Hansluwka, H.E. (1985). Measuring the health of populations, indicators and interpretations. Social science and medicine, 20(12): 1207-1224.

Hunt, S.M. et al. (1986). Measuring health status. London, Croom Helm.
Katz, S., ed. (1987). The Portugal Conference: measuring quality of life and functional status in clinical practice and epidemiological research. Journal of chronic diseases, 40, (6).

Lohr, K.N. \& Ware, J.E., Jr., ed. (1987). Proceedings of the Advances in Health Assessment Conference. Journal of chronic diseases, 40 (Suppl. No. 1).

McDowell, I. \& Newell, C. (1987). Measuring health: a guide to rating scales and questionnaires. New York, Oxford University Press.

Mootz, M. (1986). Health indicators. Social science and medicine, 22(2): 255-263.

Noack, H. (1987). Concepts of health and health promotion. In: Abelin, T. et al., ed. Measurement in health promotion and protection. Copenhagen, WHO Regional Office for Europe (WHO Regional Publications, European Series, No. 22).

Ottawa Charter for Health Promotion (1986). Health promotion, 1(4): iii-v.

Read, J.L. et al. (1987). Measuring overall health: an evaluation of three important approaches. Journal of chronic diseases, 40 (Suppl. No. 1): 78-218.

WHO (1981). Development of indicators for monitoring progress towards health for all by the year 2000. World Health Organization, Geneva ("Health For All" Series, No. 4).

WHO (1984). Health promotion. A discussion document on the concepts and principles. Copenhagen, WHO Regional Office for Europe (unpublished document ICP/HSR 602 (m01)).

WHO (1985). Targets for health for all. Copenhagen, WHO Regional Office For Europe. 
